\title{
Valutazione di controlli di qualità per sistemi di amplificazione molecolare qualitativi e quantitativi nella ricerca di HBV-DNA, HCV-RNA, CMV-DNA, e HIV-DNA
}

\author{
Alberta Azzi', Federico Chiodo', Carlo Mancini', Paolo Ravanini', \\ Maria Luisa Zerbini', Maria Carla $\mathbf{R e}^{2}$, Maurizio Gramegna ${ }^{3}$ \\ 'Comitato per lo Studio delle Biotecnologie (CoSBio) dell'AMCLI \\ ${ }^{2}$ Sezione di Microbiologia del Dipartimento di Medicina Clinica Specialistica e Sperimentale, Policlinico S. Orsola di Bologna \\ ${ }^{3} \mathrm{CLONIT} \mathrm{SrI}$
}

Key words: Quality control, amplification assay, molecular diagnosis, standardization

\author{
Characteristics of quality controls for qualitative and quantitative assays for \\ HBV - DNA, HCV - RNA, CMV - DNA and HIV - DNA
}

\section{SUMMARY}

NAT (Nucleic Acid Amplification Technology) assays are now widely used in diagnostic microbiology. The implementation of NAT assays requires the use and the availability of control reagents, with various aims, such as to verify the efficiency of the procedures and the comparability of the results obtained among different laboratories. At this purpose, the Committee for the Study of Biotechnologies (CoSBio) of AMCLI performed a study to evaluate a group of synthetic quality controls for the detection of HBV-DNA, HCV-RNA, HIV-DNA e CMV-DNA, commercially distributed by AlfaWassermann. Here we report the results of the first part of the study performed to assess the main characteristics of these reagents and their applicability to different diagnostic systems. In the second part, the same controls will be evaluated in a national multicentre study.

\section{INTRODUZIONE}

L'impiego di tecnologie di vario tipo per l'amplificazione degli acidi nucleici (NAT), si è ormai ampiamente diffuso ed affermato in molti settori della diagnostica microbiologica e virologica. Ai saggi di tipo qualitativo si affiancano sempre più frequentemente test quantitativi, oggi essenziali nel monitoraggio delle infezioni e delle terapie (1, $2,5,8)$.

Recentemente, a scopo diagnostico, sono stati introdotti numerosi metodi sia di tipo commerciale che "home made", basati su NAT, come la RTPCR, la nested-PCR, la branched-DNA, la RealTime PCR e la PCR-competitiva.

Tutti questi metodi analitici sono calibrati su propri standards e pertanto possono presentare caratteristiche di sensibilità e di specificità differenti.

È quindi chiaro che gli stessi campioni analizzati con test di tipo diverso possono dare risultati non pienamente sovrapponibili, ma è anche noto che gli stessi campioni analizzati in laboratori diversi possono dare risultati diversi anche se analizzati con lo stesso tipo di test e che, nello stesso laboratorio, il medesimo test, utilizzato in momenti diversi, può avere una efficienza diversa. Da qui la necessità di controlli per la standardizzazione, cioè di controlli di qualità esterni da utilizzare sia per valutare la piena efficienza del laboratorio sia per garantire la possibilità di confrontare risultati ottenuti in laboratori diversi.

L'importanza della standardizzazione dei metodi basati su NAT, oramai di largo impiego nei laboratori diagnostici di microbiologia e virologia, è sottolineata da numerosi studi sia a livello nazionale che a livello europeo $(11,12)$.

Da queste ricerche è risultato chiaro che l'utilizzo regolare di controlli o standard di riferimento migliora notevolmente la qualità dei risultati ottenuti dai laboratori diagnostici, sia come sensibilità che come specificità (9).

A tal fine è determinante l'affidabilità dei reagenti da utilizzare come controlli esterni. Molti studi sono stati effettuati per definire degli standard internazionali utilizzabili dai vari laboratori per rendere più omogenei $\mathrm{i}$ risultati, in particolare quelli ottenuti da metodi NAT di tipo quantitativo. Per alcuni patogeni soltanto, quali HCV, HIV, HBV e parvovirus B19, esistono oggi standard internazionali, contenenti un numero definito di unità internazionali $(10,12)$. 
Tali reagenti vengono utilizzati in indagini multicentriche, sia a livello nazionale che internazionale, come controlli di qualità esterni, ma la loro disponibilità, per quanto ampia, non è illimitata.

La preparazione e l'ampia disponibilità di validi controlli di qualità per saggi basati su NAT nella diagnostica microbiologica e virologica, in aggiunta $\mathrm{o}$ in alternativa agli standard internazionali, è quindi un fattore determinante per la garanzia dell'efficienza diagnostica dei laboratori.

Con questo obiettivo l'AMCLI, tramite il Comitato di Studio per le Biotecnologie (CoSBio), si è impegnata a valutare una serie di controlli di qualità messi in distribuzione da AlfaWassermann. Oggetto della valutazione sono stati i controlli di qualità per HBV-DNA, HCVRNA, HIV-DNA e CMV-DNA.

La valutazione si articola in due fasi.

Una prima fase dello studio, del quale qui si riportano i risultati, aveva lo scopo di verificare le principali caratteristiche dei controlli e la loro applicabilità a sistemi diagnostici diversi.

In una seconda fase, la validità dei controlli proposti verrà verificata in una indagine multicentrica a livello nazionale.

\section{MATERIALI E METODI \\ Controlli}

HBV DNA (genoma completo). Il genoma completo è clonato in un plasmide, purificato mediante centrifugazione in gradiente di cloruro di cesio. Buffer: 10mM Tris pH8.0, 1 mM EDTA.

CMV DNA (regione IEA). La regione IEA di CMV è clonata in plasmide, purificato mediante centrifugazione di cloruro di cesio.

Buffer: $10 \mathrm{mM}$ Tris $\mathrm{pH}$ 8.0, $1 \mathrm{mM}$ EDTA.

HCV RNA sintetico (regione 5'UTR). RNA sintetico di HCV-regione 5'UTR: Buffer EDTA, sodio azide $0,05 \%$, Poly-A in acqua DEPC.

HIV DNA (regione gag).

La regione gag di HIV è clonata in un plasmide, purificato mediante centrifugazione in gradiente di cloruro di cesio. Buffer: 10mM Tris pH8.0, 1 mM EDTA.

I controlli sono stati forniti dalla Soc. AlfaWassermann a quattro laboratori afferenti al Comitato di Studio per le Biotecnologie (CoSBio) dell'AMCLI, secondo uno schema concordato: due laboratori, il laboratorio di Microbiologia del Policlinico Umberto I di Roma e il Laboratorio di Microbiologia dell'A. Ospedale Maggiore della Carità di Novara sono stati incaricati di valutare i controlli per $\mathrm{HCV}$ RNA e per HBV-DNA; il laboratorio di Microbiologia e Virologia dell'Ospedale Niguarda Cà Granda di Milano è stato incaricato della valutazione dei controlli per CMV-DNA, mentre la valutazione dei controlli per HIV-DNA è stata affidata alla Sezione di Microbiologia del Dipartimento di Medicina Clinica Specialistica e Sperimentale, Policlinico S.Orsola di Bologna.

I protocolli di valutazione dei controlli prevedevano l'uso ripetuto dei controlli stessi in diverse serie di prove condotte utilizzando campioni clinici preselezionati, in test commerciali, per quanto riguarda HCV-RNA, HBVDNA e CMV-DNA, e in test "home made" per HIV-DNA.

\section{Saggi utilizzati}

Per la determinazione qualitativa di HCV-RNA è stato utilizzato il sistema Cobas Amplicor Monitor HCV qualitativo (Roche), mentre la determinazione quantitativa di HCV-RNA è stata eseguita mediante l'utilizzo del sistema Versant HCV RNA 3.0 - bDNA (Bayer).

Per le determinazioni qualitative di HBV-DNA è stato impiegato un metodo PCR-Nested di Amplimedical; per le determinazioni quantitative sono stati utilizzati il sistema Versant-Bayer e il Cobas Amplicor Monitor HBV-Roche.

Per il CMV-DNA sono stati utilizzati un metodo qualitativo basato su nested-PCR e, come test quantitativo, il metodo Ibridoquantum, entrambi di Amplimedical.

Per HIV-DNA sono stati utilizzati metodi "home made", PCR qualitativa e PCR realtime, messi a punto presso il laboratorio di Bologna, precedentemente descritti $(3,4)$.

\section{Brevemente:}

Primers specifici per un frammento del gene gag di HIV-1: SK431:

5'-TGCTATGTCAGTTCCCCTTGGTTCTCT-3'

SK462:

5'-AGTTGGAGGACATCAAGCAGCCATGCAAAT-3'

Reagenti per Real-time: Quantitect SYBR Green

PCR kit (Qiagen) utilizzati come da istruzioni kit.

Apparecchio di amplificazione e analisi

Real-time: LightCycler Instrument Roche

Software analisi e gestione dati:

LightCycler 5.3.2

Quantità DNA dei campioni utilizzata per ogni singolo campione o replicato: $600 \mathrm{ng}$ (pari a circa 200.000 cellule). I campioni sono stati analizzati in triplicato.

Controllo positivo: Controllo di seduta (REF 059 60331) come da istruzioni e DNA estratto da cellule 8E5LAV (contenenti una copia di HIV per cellula).

Controllo negativo: DNA estratto da cellule Jurkat negative per HIV-1 in duplicato.

Curve Standard di riferimento: (da 1 copia a 100.000 copie/diluizione standard per plasmide 
Alfa-Wassermann, da 1 copia a 10.000 copie/diluizione standard per il plasmide ARP95 PCR reference kit., da 1 copia a 10.000 copie/diluizione di DNA estratto da 8E5LAV. In ogni seduta veniva determinata la curva standard su cui impostare il software per la determinazione quantitativa.

Controllo di amplificazione sui campioni clinici in parallelo: amplificazione nelle stesse condizioni con primers per la globina. 3.1 Condizioni di amplificazione e di analisi dati:

Il protocollo della reazione prevedeva 15 minuti a $95^{\circ} \mathrm{C}$, per l'attivazione della Taq polimerasi, seguiti da 45 cicli di amplificazione (Denaturazione a $94^{\circ} \mathrm{C}$ per 10 secondi, Annealing a $60^{\circ} \mathrm{C}$ per 30 secondi, Extension a $72^{\circ} \mathrm{C}$ per 30 secondi). La temperatura di analisi era di $78^{\circ} \mathrm{C}$, per 3 secondi.

I risultati quantitativi dei campioni sono stati normalizzati come copie di HIV-1 DNA $/ 1 \times 10^{6}$ cellule

\section{RISULTATI \\ Valutazione dei controlli per HCV-RNA}

\section{CONTROLLI PER TEST QUALITATIVI.}

I 2 controlli proposti per i test qualitativi contenevano $1 \times 10^{7}$ copie $/ \mu 1$ e $1 \times 10^{4}$ copie $/ \mu 1$.

Preliminarmente sono stati controllati e confermati i valori dichiarati.

I due controlli sono stati valutati in entrambi i laboratori in parallelo a 24 campioni clinici, selezionati secondo uno schema prestabilito:

- 12 campioni positivi selezionati mediante dosaggio degli anticorpi (ELISA e RIBA) e test di amplificazione

- 12 campioni negativi selezionati mediante dosaggio degli anticorpi (ELISA e RIBA) e test di amplificazione, così caratterizzati: 4 campioni negativi a tutti $\mathrm{i}$ test; 4 campioni HCVAb-ELISA positivi, HCVAb-RIBA negativi, PCR negativi; 4 campioni HCVAb-ELISA positivi, HCVAb-RIBA indeterminati, PCR negativi.

I campioni clinici hanno dato i risultati attesi ed entrambi i controlli sono risultati fortemente positivi.

\section{CONTROLLI PER TEST QUANTITATIVI.}

Per i test quantitativi sono stati forniti 4 controlli a diversa concentrazione: S1, S2, S3, S4 (Tabella 1).

I risultati delle determinazioni quantitative ottenute nei due laboratori sono riportati in Tabella 1. Considerando che nelle tecniche di amplificazione molecolare differenze all'interno di un intervallo di $+/-\log 0,5$ non sono significative, i risultati ottenuti dai due laboratori si possono conside- rare abbastanza sovrapponibili.

I controlli S1 e S2 avevano una concentrazione troppo vicina al limite massimo del range di amplificazione lineare del metodo utilizzato.

Allo scopo di determinare le concentrazioni dei controlli più adatte per $i$ test qualitativi e quantitativi attualmente più utilizzati è stata quindi analizzata, presso il laboratorio di Novara, una serie di 8 diluizioni del controllo in oggetto (da $10^{\circ}$ a $10^{7}$ ) mediante il test Cobas Amplicor HCV Roche. Le diluizioni sono state aggiunte nella procedura analitica a livello già della fase di incubazione con il reagente di lisi, utilizzando $10 \mu \mathrm{L}$ di controllo $+200 \mu \mathrm{L}$ di siero negativo.

I campioni così costituiti hanno poi seguito in tutte le fasi il procedimento analitico del metodo utilizzato.

Considerando il rapporto tra copie e U.I., il volume di campione impiegato, la quantità del campione dopo aggiunta del siero negativo $(200 \mu \mathrm{L})$, è stato calcolato che le concentrazioni saggiate andavano da 10 a $10^{8} \mathrm{UI}$ per $\mathrm{mL}$.

L'analisi di tutte le diluizioni ha dato esito positivo, ad eccezione del campione a più bassa concentrazione $\left(10^{1}\right.$ U.I. $\left./ \mathrm{mL}\right)$, che ha dato origine ad un segnale in zona grigia (gray zone).

Tuttavia, quest'ultima concentrazione era al di sotto dei valori di sensibilità dichiarata dal test utilizzato.

Sulla base di questi dati, è stata predisposta una nuova serie di controlli, che sono stati inviati al laboratorio di Roma per una ulteriore valutazione. I controlli, indicati come C-1, C-2, C-3 contenevano rispettivamente $10,100,1000$ copie di $\mathrm{HCV}$ $\mathrm{RNA} / \mu 1$.

Nella tabella 2 sono riportati i risultati della valutazione quantitativa di una nuova serie di controlli. Considerando il volume di campione impiegato e la quantità del campione dopo aggiunta del siero negativo, si può concludere che vi è la discordanza tra $\mathrm{i}$ valori attesi e quelli ottenuti è entro limiti accettabili.

I controlli sono stati poi saggiati insieme a 24 campioni clinici, secondo il seguente schema:

- 12 campioni positivi selezionati mediante dosaggio degli anticorpi (ELISA e RIBA) e test di amplificazione, comprendenti: 4 campioni con carica virale $<3200$ copie di HCVRNA/ml; - 4 campioni con carica virale compresa tra $1 \times 10^{4}$ e $50 \times 10^{4}$ copie di HCVRNA/ml; 4 campioni con carica virale $>5 \times 10^{6}$ copie di HCVRNA/ml).

- 12 campioni negativi selezionati mediante dosaggio degli anticorpi (ELISA e RIBA) e test di amplificazione.

In tutte le prove i campioni clinici e i controlli hanno dato $i$ valori aspettati. 


\section{Valutazione dei controlli per HBV-DNA}

CONTROLli PER TEST QUALITATIVI

I 2 controlli proposti per i test qualitativi contenevano rispettivamente $1 \times 10^{7} \mathrm{copie} / \mu \mathrm{l}$ e $1 \times 10^{4} \mathrm{copie} / \mu 1$. Valutati, preliminarmente con il metodo quantitativo (usando $10 \mu \mathrm{l}$ di controllo portati a $200 \mu \mathrm{l}$ con siero negativo) il risultato ottenuto è stato di > 100.000.000 copie/ml e $96.120 \mathrm{copie} / \mathrm{ml}$, rispettivamente. La concentrazione nel primo controllo è risultata troppo elevata.

I controlli sono stati poi saggiati insieme ai campioni clinici secondo il seguente schema:

- 12 campioni positivi selezionati mediante studio dei markers dell'epatite B (6 HBeAg negativi6 HBeAg positivi B) e nested-PCR

- 12 campioni negativi selezionati mediante studio dei markers epatite B e nested-PCR (comprendenti: 8 campioni negativi a tutti $\mathrm{i}$ test e - 4 campioni HBcAb positivi, PCR negativi)

Tutti i campioni clinici utilizzati nelle prove, come da protocollo, hanno dato i risultati attesi e i controlli sono risultati fortemente positivi.

\section{CONTROLli PER TEST QUANTITATIVI}

Per la valutazione quantitativa sono stati forniti 4 controlli a diversa concentrazione $\left(\mathrm{S} 1: 1 \times 10^{6}\right.$, $\mathrm{S} 2: 1 \times 10^{5}, \mathrm{~S} 3: 1 \times 10^{4}, \mathrm{~S} 4: 1 \times 10^{3}$ )

I risultati dell'analisi quantitativa di questi controlli sono riportati in tabella 3 .

Con il sistema Versant-Bayer, forse per il suo più ampio intervallo di amplificazione lineare, le copie /ml ottenute erano più corrispondenti a quelle ipotetiche, dopo diluizione dei controlli secondo le necessità del sistema analitico utilizzato.

I controlli sono stati saggiati insieme a:

- 12 campioni positivi selezionati mediante studio dei markers dell'epatite B e test di amplificazione (comprendenti: 4 campioni con carica virale $<2000$ copie di HBVDNA/ml; 4 campioni con carica virale compresa tra $5 \times 10^{3}$ e

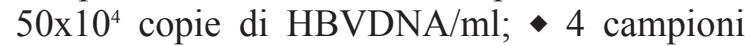
con carica virale $>20 \times 10^{6} \mathrm{HBVDNA} / \mathrm{ml}$ );

- 12 campioni negativi selezionati mediante studio dei markers epatite B e test di amplificazione.

In tutti i casi i campioni clinici hanno dato i risultati attesi e i controlli hanno confermato $i$ risultati riportati nella tabella 3 .

\section{Valutazione di controlli per CMV-DNA}

Sono stati forniti controlli a due concentrazioni: L- 1 X10 ${ }^{4}$ copie $/ \mathrm{ml}$ e H-1X10 ${ }^{7}$ copie/ml.

Entrambi gli standard sono stati testati in triplo in 5 sedute di lavoro comprendenti campioni (sangue intero o plasma) risultati positivi o negativi:

- 12 campioni positivi selezionati mediante dosaggio anticorpi IgG/IgM (ELISA) e CMV IEA (pp65) e
- 12 campioni negativi selezionati mediante come sopra riportato (comprendenti: 4 campioni negativi a tutti i test e $\bullet$ campioni CMV IgG positivi, CMV IgM negativi, CMV IEA (pp65) negativi).

Lo standard L ha fornito risultati molto riproducibili nell'ordine di grandezza di $3 \times 10^{4}$.

Lo standard $\mathrm{H}$ ha fornito risultati superiori rispetto al range dosabile con la metodica in uso.

Tutti $\mathrm{i}$ campioni clinici hanno dato $\mathrm{i}$ risultati aspettati.

\section{Valutazione dei controlli per HIV-DNA.}

I controlli forniti sono stati saggiati secondo il protocollo prestabilito che prevedeva l'analisi di campioni selezionati in triplicato insieme ai controlli di qualità proposti, sia nei saggi qualitativi che in quelli quantitativi, al fine di poter valutare la riproducibilità dei risultati e la corrispondenza all'attesa dei livelli degli standard proposti.

Per la determinazione qualitativa di HIV-1 DNA sono stati utilizzati:

- 12 Campioni positivi, precedentemente determinati mediante dosaggio degli anticorpi (ELISA e WB) e PCR-DNA e

- 12 Campioni negativi, precedentemente determinati mediante dosaggio degli anticorpi (ELISA e WB) e PCR-DNA ( 4 completamente negativi a tutti $\mathrm{i}$ test precedentemente menzionati; 4 provenienti da soggetti adulti, con almeno un risultato sierologico indeterminato, ma negativi mediante reazione di amplificazione a catena; 4 provenienti da neonati, di età compresa tra 0 e 8 mesi, partoriti da madri HIV1 infette, positivi ai test sierologici ma negativi mediante reazione di amplificazione a catena).

Per la determinazione quantitativa di HIV-1 DNA sono stati utilizzati:

- 12 campioni positivi, precedentemente determinati mediante dosaggio degli anticorpi (ELISA e WB) e PCR-DNA ( 4 con carica virale plasmatica inferiore a 50 copie di HIV-1 RNA/ml, - 4 con carica virale plasmatica compresa tra $1 \times 10^{2}$ e $1 \times 10^{4}$ copie di HIV-1 RNA/ml, 4 con carica virale plasmatica superiore a $1 \times 10^{4}$ copie di HIV-1 RNA/ml) e

- 12 campioni negativi, precedentemente determinati mediante dosaggio degli anticorpi (ELISA e WB) e PCR-DNA $(\bullet 4$ completamente negativi a tutti $\mathrm{i}$ test precedentemente menzionati; 4 provenienti da soggetti adulti, con almeno un risultato sierologico indeterminato, ma negativi mediante reazione di amplificazione a catena; 4 provenienti da neonati di età compresa tra 0 e 8 mesi, partoriti da madri HIV1 infette, positivi ai test sierologici ma negativi mediante reazione di amplificazione a catena). 
Nella Tabella 4 sono riportati i risultati dell'analisi quantitativa dei 12 campioni positivi, usando come calibratori sia i controlli oggetto dello studio che un altro plasmide in uso presso il laboratorio. Il numero di copie di DNA è stato determinato facendo una media dei valori ottenuti all'interno della stessa seduta e tra le tre sedute differenti, considerando che per ogni seduta i campioni venivano testati in triplicato.

I valori sono stati normalizzati su un milione di cellule (PBMC).

Inoltre, in tutti i campioni provenienti dai soggetti non infetti non è stato possibile osservare nessun segnale positivo

\section{DISCUSSIONE}

Attualmente le tecniche NAT sono ampiamente utilizzate in tutti i laboratori diagnostici di microbiologia clinica e, specialmente le tecniche quantitative, sono considerate fondamentali nella valutazione di un processo infettivo anche ai fini dell'applicazione della terapia.

Molti lavori sono stati sviluppati al fine di definire i valori quantitativi delle cariche microbiche, registrate da questi metodi, correlabili con uno stato di malattia o per impostare una terapia.

I dati che vengono forniti dai metodi NAT hanno spesso un valore clinico molto elevato e pertanto richiedono un alto indice di standardizzazione e di controllo.

Molti studi sono stati effettuati, sia a livello nazionale che internazionale, allo scopo di valutare, migliorare o mantenere elevata la qualità delle prestazioni dei laboratori, ma anche di confrontare e valutare le tecniche diagnostiche.

In due recenti studi condotti dal CoSBio $(6,7)$ per la standardizzazione di metodi NAT qualitativi e quantitativi per la ricerca di HCV-RNA, è emersa chiaramente l'importanza dell'utilizzo di standard o controlli esterni e inoltre è stato messo in evidenza che, complessivamente, la sensibilità ottenuta dai laboratori partecipanti per quanto riguarda la diagnostica qualitativa di HCV-RNA, era passata, nel secondo studio, dal 95, 65 al 99,5\%.

Questi studi hanno anche evidenziato che, particolarmente per la standardizzazione dei metodi quantitativi, i controlli migliori erano quelli che avevano una carica genomica all'interno del valore ideale medio del range di amplificazione lineare del metodo NAT utilizzato (circa $\left.10^{4} / 10^{5}\right)$, mentre per quelli che vengono definiti "run control" o controlli di amplificazione potevano essere utilizzati mediamente a concentrazioni inferiori di un log.

Essenziale è quindi la disponibilità di standard o controlli di qualità da utilizzare con regolari- tà in fasi diverse delle procedure di controllo di qualità.

Gli standard internazionali sono certamente i più validi controlli di riferimento, ma per ora ne esistono solo per pochi patogeni e la loro disponibilità non è illimitata.

Occorrono quindi altri reagenti, working reagents, appunto, da utilizzare più comunemente $\mathrm{e}$ più frequentemente, anche per patogeni meno diffusi o emergenti.

Il controllo di tali reagenti deve essere tuttavia rigoroso perché dalla loro validità può dipendere la validità delle procedure diagnostiche impiegate. In questo studio il CoSBio ha condotto una valutazione preliminare di controlli di qualità proposti dalla Ditta Alfa-Wassermann per l'HCV-RNA, l'HBV-DNA, il CMV-DNA e l'HIV-DNA sia in sistemi qualitativi che in sistemi quantitativi.

Questi controlli sono stati valutati da alcuni laboratori con una buona esperienza nel campo della diagnostica molecolare, utilizzandoli insieme a campioni clinici preselezionati in saggi analitici di grande diffusione nei laboratori a grande campionatura giornaliera.

Una prima serie di prove ha messo in evidenza che $i$ controlli in oggetto mostravano una buona robustezza, ma, sia quelli per HCV-RNA e HBVDNA che quelli per CMV DNA erano forniti a concentrazioni troppo elevate mentre risultava più opportuno inserire controlli a concentrazioni più vicine ai limiti di sensibilità dei metodi di amplificazione per cui sono proposti.

I controlli proposti per HIV-DNA, valutati con una tecnica real-time PCR, sono sembrati ben equilibrati.

In effetti, tutti i controlli in oggetto erano stati calibrati essenzialmente per essere utilizzati con metodiche di real-time PCR, che sembrano avere una calibratura più elevata rispetto alle metodiche oggi maggiormente utilizzate, quali la BranchedDNA e la PCR competitiva.

Da una seconda serie di prove è emerso che alcuni di questi problemi sono stati eliminati e nel formato riproposto le concentrazioni sono risultate tali da poter risultare efficaci per il controllo di amplificazione della maggioranza delle metodiche in uso.

Dopo questa preliminare valutazione, i controlli proposti dovranno essere valutati in indagini policentriche con la partecipazione di un sufficiente numero di laboratori di buon livello.

In indagini di questo tipo vengono scelti anche gli standard internazionali e gli working reagents. Sono queste indagini che possono mettere in evidenza eventuali difetti o limiti e suggerire possibili correttivi, o possono semplicemente confermare la validità di un prodotto. 
Tabella I. Valutazione quantitativa dei controlli per HCV-RNA

\begin{tabular}{llll}
\hline Controllo & Valore ipotetico* & Lab Roma** & Lab Novara** \\
\hline SI & $2 \times 10^{8}$ copie $/ \mathrm{ml}$ & $>40.000 .000$ copie $/ \mathrm{ml}$ & $>40.000 .000 \mathrm{copie} / \mathrm{ml}$ \\
\hline S2 & $2 \times 10^{7} \mathrm{copie} / \mathrm{ml}$ & $>40.000 .000 \mathrm{copie} / \mathrm{ml}$ & $>40.000 .000 \mathrm{copie} / \mathrm{ml}$ \\
\hline S3 & $2 \times 10^{6} \mathrm{copie} / \mathrm{ml}$ & $10.126 .812 \mathrm{copie} / \mathrm{ml}$ & $4.426 .435 \mathrm{copie} / \mathrm{ml}$ \\
\hline S4 & $2 \times 10^{5} \mathrm{copie} / \mathrm{ml}$ & $1.060 .517 \mathrm{copie} / \mathrm{ml}$ & $375.385 \mathrm{copie} / \mathrm{ml}$ \\
\hline
\end{tabular}

*concentrazioni ipotetiche dei controlli, dopo estrazione; ${ }^{* *}$ media valori su tre prove

Tabella 2. II valutazione quantitativa dei controlli per HCV-RNA

\begin{tabular}{|c|c|c|}
\hline Controllo & Valore atteso (copie/ml) & Risultato (copie/ml) * \\
\hline $\mathrm{C}-\mathrm{I}$ & 10.000 & 70.000 \\
\hline $\mathrm{C}-2$ & 100.000 & 700.000 \\
\hline $\bar{C}-3$ & 1.000 .000 & 7.000 .000 \\
\hline
\end{tabular}

*valore medio delle prove

Tabella 3. Valutazione quantitativa dei controlli per HBV

\begin{tabular}{|c|c|c|c|}
\hline controllo & Valore ipotetico & Versant-Bayer & COBAS Amplicor \\
\hline SI & $\mid \times 10^{8}$ copie $/ \mathrm{ml}$ & $>|x| 0^{8}$ copie $/ \mathrm{ml}$ & $>200.000$ copie $/ \mathrm{ml}$ \\
\hline $\mathrm{S2}$ & IxI07 copie/ml & I7.022.742 copie/ml & $>200.000$ copie/ml \\
\hline S3 & $\mathrm{I} \times 10^{6} \mathrm{copie} / \mathrm{ml}$ & I.II5.450 copie/ml & I20.000 copie/ml \\
\hline S4 & $\mathrm{I} \times 10^{5} \mathrm{copie} / \mathrm{ml}$ & I33.8I4 copie/ml & 19.000 copie/ml \\
\hline
\end{tabular}

Tabella 4. Valutazione quantitativa controlli HIV-RNA e campioni clinici

\begin{tabular}{lcccc}
\hline Campioni & $\begin{array}{c}\text { Viral-load RNA } \\
\text { (copie/ml) }\end{array}$ & $\begin{array}{c}\text { Viral-load DNA (copie/I } \times 10^{6} \text { cell.) } \\
\text { Alfa-Wasserman }\end{array}$ & $\begin{array}{c}\text { Viral-load DNA (copie/I } \times 10^{6} \mathrm{cell} \text {.) } \\
\text { plasmide ARP956 }\end{array}$ & $\begin{array}{c}\Delta s \\
\text { log } 10\end{array}$ \\
\hline 1 & $<50$ & $8 \times 10^{1}(1.90)$ & $1.6 \times 10^{2}(2.20)$ & 0.3 \\
\hline 2 & $<50$ & $1.8 \times 10^{4}(4.26)$ & $7.6 \times 10^{3}(3.88)$ & 0.38 \\
\hline 3 & $<50$ & $9.2 \times 10^{3}(3.96)$ & $3.6 \times 10^{3}(3.55)$ & $0.4 \mathrm{I}$ \\
\hline 4 & $<50$ & $7.2 \times 10^{2}(2.86)$ & $1.9 \times 10^{2}(2.69)$ & 0.17 \\
\hline 5 & $1.4 \times 10^{2}$ & $1.6 \times 10^{3}(3.20)$ & $9.3 \times 10^{2}(2.97)$ & 1.0 \\
\hline 6 & $1.0 \times 10^{4}$ & $8.9 \times 10^{2}(2.95)$ & $9.0 \times 10^{4}(4.95)$ & 0.02 \\
\hline 7 & $3.8 \times 10^{3}$ & $8.5 \times 10^{4}(4.92)$ & $4.0 \times 10^{3}(3.60)$ & 0.03 \\
\hline 8 & $6.6 \times 10^{3}$ & $3.4 \times 10^{3}(3.53)$ & $1.0 \times 10^{3}(3.0)$ & 0.07 \\
\hline 9 & $4.6 \times 10^{5}$ & $3.8 \times 10^{2}(2.58)$ & $1.5 \times 10^{2}(2.18)$ & 0.42 \\
\hline 10 & $>500000$ & $2.2 \times 10^{2}(2.34)$ & $2.4 \times 10^{2}(2.38)$ & 0.16 \\
\hline 11 & $1.2 \times 10^{5}$ & $1.9 \times 10^{2}(2.27)$ & $3.8 \times 10^{2}(2.57)$ & 0.11 \\
\hline 12 & $1.9 \times 10^{5}$ & & & 0.33 \\
\hline
\end{tabular}




\section{BIBLIOGRAFIA}

1. Berger AM, Preiser W. Viral genome quantification as a tool for improving patient management: the example of HIV, HBV, HCV and CMV. J Antimicrob Chemother 2002; 49: 713-21.

2. Fernet G. Molecular diagnostics in virology. J Clin Virol 2004; 31: 239-47.

3. Gibellini D, Vitone F, Gori E, et al. Quantitative detection of human immunodeficiency virus type 1 (HIV-1) viral load by SYBR green real-time RT-PCR technique in HIV-1 seropositive patients. J Virol Methods 2004; 115: 183-9.

4. Gibellini D, Vitone F, Schiavone P, et al. Quantitative detection of human immunodeficiency virus type 1 (HIV-1) proviral DNA in peripheral blood mononuclear cells by SYBR green real-time PCR technique. J Clin Virol. 2004; 29: 282-9.

5. Mackay IM. Real-time PCR in the microbiology laboratory. Clin Microbiol Infect 2004; 10: 190-212.

6. Mancini C, Pisani G, Azzi A, et al. Inter-laboratory comparison of qualitative and quantitative detection of hepatitis $\mathrm{C}(\mathrm{HCV})$ virus RNA in diagnostic virology: a multicentre study (MS) in Italy. J Clin Virol 2004; 30: 313-9.

7. Mancini C, Zerbini M, Azzi A. et al. Multicentre Italian Study Group (MISG) for the standardization of hepatitis C virus (HCV) PCR. J Clin Virol. 2003; 27 : 83-9.

8. Niesters HGM. Molecular and diagnostics clinical virology in real time. Clin Microbiol Infect 2004; 10: 5-11.

9. Pisani G, Cristiano K, Saldanha J, et al. External qualità assessment for the detection of blood-borne viruses in plasma by nucleic acid amplification technology: the first human immunodeficiency virus and hepatitis B virus studies (HIV EQA/1 and HBV EQA/1) and the fifth hepatitis $\mathrm{C}$ virus study (HCV EQA/5). Vox Sanguinis 2004; 87: 91-5.

10. Saldanha J, Lelie N, Yu MW, et al. Establishment of the first World Health Organization ternational Standard for human parvovirus B19 DNA nucleic acid amplification techniques. Vox Sanguinis 2002; 82: 24-31.

11. Saldanha J. Standardization: a Progress Report. Biologicals 1999; 27: 285-9.

12. Saldanha J. Validation and standardization of nucleic acid amplification technology (NAT) assay for the detection of viral contamination of blood and blood products. J Clin Virol 2001; 20: 7-13.

Alberta Azzi

Dipartimento di Sanità Pubblica Viale Morgagni 48, 50134 Firenze Tel 055 4598557; Fax 0554598924 E-mail: alberta.azzi@unifi.it 